\title{
The Set of Poles of a Two-Sheeted Hyperboloid
}

\author{
Robert Sinclair and Minoru Tanaka
}

\section{CONTENTS}

1. Introduction

2. Algorithm

3. Analysis

4. Independent Checks

5. Results for the Two-Sheeted Hyperboloid

6. Conclusion

Acknowledgements

References
2000 AMS Subject Classification: Primary, Secondary

Keywords: mathematics, function, equation
It has been conjectured for some time that the set of poles of a rotationally symmetric two-sheeted hyperboloid breaks into two disjoint sets if symmetry is broken by contraction perpendicular to the original axis of symmetry. We provide the first reliable visualizations of this process, confirming previous conjectures and motivating new ones.

\section{INTRODUCTION}

Despite its being a branch of geometry, there are surprisingly few visualizations of the objects or results of study of global differential geometry, since even approximate computations are difficult due to their global nature. See [Berger, 00] for a recent historical survey.

Poles are points whose cut-locus is void, but explicit cut-loci are essentially intractable by pure mathematics today. For example, it is still not known whether the cutloci of ellipsoids, even of revolution, are all topological segments.

This was stated in [von Braunmühl, 1882], which is an extract, with various extensions, of an earlier paper which appeared in 1878. The problem is that von Braunmühl was using Jacobi's last "theorem", still not proved (see for example page 39 of [Arnold, 1994]). Jacobi's statement is linked with many other topics.

The only known cut-loci are for compact symmetric spaces, one kind of homogeneous metric on the threedimensional sphere and von Mangoldt surfaces of revolution (which include paraboloids and two-sheeted hyperboloids) [Tanaka, 92a]. The field is a very natural one, concerning the uniqueness of shortest connections between points.

In 1880, Hans von Mangoldt wrote a paper with the title "About those Points on Positively Curved Surfaces which have the Property that the Geodesic Curves proceeding from them never cease to be Shortest" [von Mangoldt, 1881]. This was a continuation of a line of inquiry begun by Jacobi, and subsequently worked on by others 
such as von Braunmühl, as mentioned above. Von Mangoldt's paper includes proofs of many statements concerning such points, which we call poles, for paraboloids and two-sheeted hyperboloids. In particular, he described qualitatively how the set of poles of a hyperboloid changes as it is compressed in one direction, destroying rotational symmetry. See [Tanaka, 92a] and references therein for a review of more recent work in this area.

We consider the family of two-sheeted hyperboloids (we consider only one sheet) given by the parametrization

$$
z=\sqrt{1+p x^{2}+y^{2}} .
$$

It will be convenient to give the point of intersection of these surfaces with the $z$-axis a name, so we will call the point $(x, y, z)=(0,0,1)$ the Scheitel, the German name von Mangoldt gave this point. An umbilic point is a point on the surface at which the two principal curvatures are equal. For $p=1$, the Scheitel is the only umbilic point. For $0<p<1$ and $p>1$, there are two umbilic points.

Von Mangoldt made the following two statements about the set of poles of two-sheeted hyperboloids:

1. The set of poles of a rotationally symmetric twosheeted hyperboloid includes the Scheitel of the surface, and is bounded by a circle centred at this point.

2. If one breaks rotational symmetry by making one of the equal axes smaller, then the set of poles tightens around the Scheitel until the set of poles breaks into two disjoint sets, each containing an umbilic point.

The surface given by $(1-1)$ is rotationally symmetric for $p=1$. The process of making one of the equal axes shorter described by von Mangoldt corresponds to making $p$ ever greater than 1 . Values of $p$ less than 1 correspond to a hyperboloid stretched in one direction. Note that for $p=0$, the surface is flat and all points are poles.

Von Mangoldt did not prove the second statement, only that the umbilic points are always poles and that the Scheitel ceases to be one for sufficiently large deformations of the surface (for sufficiently large values of $p$ ). We wish to make a contribution to the understanding of this process in the form of experimental data. It is hoped that proofs motivated by our visualizations will appear in the future.

\section{ALGORITHM}

The projection of any point on the surface given by (1-1) onto the $(x, y)$-plane is unique, and we will use $x$ and $y$
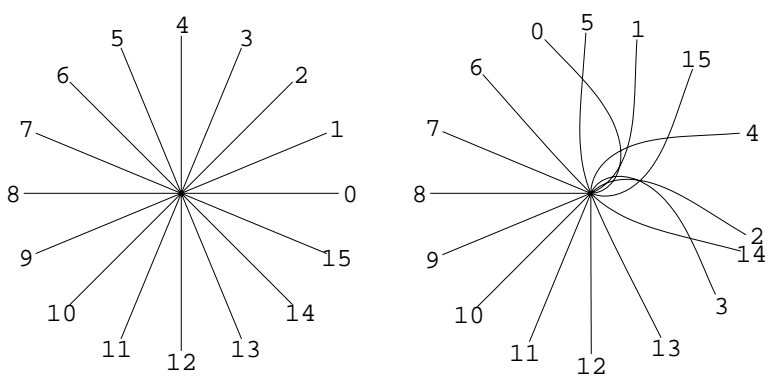

FIGURE 1. The pole identification algorithm. The point on the left is a pole: the geodesic curves proceeding from it have a fixed radial ordering $(0,1, \ldots, 14,15)$. The point on the right is not a pole, since the geodesics' ordering changes (to $0,6,7, \ldots, 12,13,3,14,2,4,15,1,5$ ).

as coordinates in the following. The geodesic equations of the surface are

$$
\left[\begin{array}{l}
x^{\prime \prime} \\
y^{\prime \prime}
\end{array}\right]=f_{p}\left(x, x^{\prime}, y, y^{\prime}\right) \cdot\left[\begin{array}{r}
p \cdot x \\
y
\end{array}\right]
$$

where differentiation is with respect to arc length $s$ and

$f_{p}\left(x, x^{\prime}, y, y^{\prime}\right)=\frac{\left(p x x^{\prime}+y y^{\prime}\right)^{2}-\left(p x^{\prime 2}+y^{\prime 2}\right)\left(1+p x^{2}+y^{2}\right)}{\left(1+p x^{2}+p^{2} x^{2}+2 y^{2}\right)\left(1+p x^{2}+y^{2}\right)}$.

These equations are numerically well-behaved, in particular because a hyperboloid is asymptotically flat, meaning that $f_{p}$ rapidly goes to zero as $x^{2}+y^{2}$ increases. Their numerical analysis is routine and will not be discussed here. As we will see, there are geometrical sources of error which are more difficult to control.

The algorithm is quite simple. A pole is a point on a surface for which none of the geodesic curves proceeding from it intersect. If such geodesic curves intersect, then there will be a change in their radial ordering (see Figure 1), and the point can be labeled as not being a pole. Otherwise the point is labeled as being a pole.

We compute a large number $(N)$ of geodesic curves all beginning at the candidate pole, with initial angles (measured in their projection onto the $(x, y)$-plane) 0 , $2 \pi / N, 4 \pi / N, \ldots,(N-1) 2 \pi / N$. These geodesic curves are all computed up to the large but finite length $d_{\max }$. We then compute the angles of their endpoints with respect to the origin (which will be close to the candidate point if $d_{\max }$ is truly large) and thereby their radial ordering.

The algorithm is correct when both $N$ and $d_{\max }$ become infinite. Errors occur when two geodesic curves from a candidate point do intersect, but at a distance greater than $d_{\max }$, or when those geodesic curves computed all intersect an even number of times, such that 
their radial ordering appears to have been conserved. In both cases, a point which is actually not a pole is labeled as being one.

Unless otherwise stated, all computations have been performed with $N=8192$ and $d_{\max }=10^{50}$.

\section{ANALYSIS}

Let $(M, g)$ be a surface of revolution homeomorphic to $\mathbb{R}^{2}$, i.e., a complete smooth Riemannian manifold homeomorphic to $\mathbb{R}^{2}$ which admits a point $p$ such that the Gaussian curvature of $(M, g)$ is constant on $S_{p}(t):=\{q \in$ $M ; d(p, q)=t\}$ for each $t>0$, where $d$ denotes the Riemannian distance function of $(M, g)$. The point $p$ on $M$ is called the vertex of $M$. The Riemannian metric $g$ can be written as

$$
g=d r^{2}+m(r)^{2} d \theta^{2}
$$

in geodesic polar coordinates $(r, \theta)$ around the vertex. The function $m:(0, \infty) \rightarrow(0, \infty)$ is equal to

$$
m(r(q))=\sqrt{g_{q}\left(\frac{\partial}{\partial \theta}, \frac{\partial}{\partial \theta}\right)}
$$

for each $q \in M \backslash\{p\}$ and extensible to a smooth odd function on $\mathbb{R}$ with $m^{\prime}(0)=1$. From now on we assume that $\liminf _{t \rightarrow \infty} m(t)$ is non-zero. Hence there exists a positive number $a_{1}$ such that $m^{\prime}>0$ on $\left[0,2 a_{1}\right], m\left(2 a_{1}\right)=$ $\inf \left\{m(t) ; t \geq 2 a_{1}\right\}$. Let $f:\left[0, m\left(2 a_{1}\right)\right] \rightarrow \mathbb{R}$ be the inverse function of $\left.m\right|_{\left[0,2 a_{1}\right]}$. Then there exists a smooth function $h$ on $\left[0, m\left(2 a_{1}\right)\right]$ satisfying $f^{\prime}(t)=1+t^{2} h(t)$. For each point $q \in M \backslash\{p\}$, let $\tau_{q}:[0, \infty) \rightarrow M$ denote the unit speed geodesic emanating from $q=\tau_{q}(0)$ with $\tau_{q}(d(p, q))=p$. The following theorem was proved in [Tanaka, 92b] (see (1.20), (1.21) and (2.6).

Theorem 3.1. Let $\left(M, d r^{2}+m(r)^{2} d \theta^{2}\right)$ denote a surface of revolution with vertex $p$, where $(r, \theta)$ denotes geodesic polar coordinates around $p$. Suppose that $\liminf _{t \rightarrow \infty} m(t)$ is non-zero. Then for each point $q \in M \backslash\{p\}$, a (non-zero) Jacobi field $Y(t)$ along $\tau_{q}(t)$ with $Y(0)=0$ is given by

$$
\begin{aligned}
Y(t) & =\left(2\left(-\frac{1}{m\left(a_{1}\right)}+m\left(a_{1}\right) h\left(m\left(a_{1}\right)\right)-\int_{0}^{m\left(a_{1}\right)} t h^{\prime}(t) d t\right)\right. \\
& \left.+\left(\int_{a_{1}}^{\rho} \frac{1}{m(r)^{2}} d r+\int_{a_{1}}^{t-\rho} \frac{1}{m(r)^{2}} d r\right)\right)\left(\frac{\partial}{\partial \theta}\right)_{\tau_{q}(t)}(3-3)
\end{aligned}
$$

for each $t>\rho:=d(p, q)$. Furthermore, if $\int_{1}^{\infty} \frac{1}{m(r)^{2}} d r$ is finite, then

$Y(t)=(2 \pi)^{2}\left(c(L)-\int_{\rho}^{\infty} \frac{1}{L^{2}(r)} d r-\int_{t-\rho}^{\infty} \frac{1}{L^{2}(r)} d r\right)\left(\frac{\partial}{\partial \theta}\right)_{\tau_{q}(t)}$

where

$$
c(L)=4 \int_{0}^{\infty} \frac{L(t)-t L^{\prime}(t)}{L(t)^{3}} d t
$$

and

$$
L(t)=2 \pi m(t)
$$

Let $i(q)$ denote the injectivity radius of $q \in M$ (see [Chavel, 93]).

Corollary 3.2. Let $p$ be the vertex of the paraboloid $z=$ $x^{2}+y^{2}$. Then

$$
\lim _{q \rightarrow p} d(p, q) \ln i(q)=1 .
$$

Proof: Let $q$ be any point on the paraboloid distinct from $p$. Then $i(q)$ satisfies

$$
Y(i(q))=0,
$$

where $Y(t)$ denotes the Jacobi field in Theorem 3.1 (see [Tanaka, 92b]). Hence we have

$$
\begin{gathered}
2\left(-\frac{1}{m\left(a_{1}\right)}+m\left(a_{1}\right) h\left(m\left(a_{1}\right)\right)-\int_{0}^{m\left(a_{1}\right)} t h^{\prime}(t) d t\right) \\
+\int_{a_{1}}^{\rho} \frac{1}{m(r)^{2}} d r+\int_{a_{1}}^{i(q)-\rho} \frac{1}{m(r)^{2}} d r=0 .
\end{gathered}
$$

Here $m(r)$ satisfies the differential equation

$$
\begin{aligned}
& m^{\prime}(r)= \frac{1}{\sqrt{1+4 m(r)^{2}}} \\
& \text { with initial condition } \quad m(0)=0
\end{aligned}
$$

in our case. Hence by l'Hopital's rule, we get

$$
\lim _{r \rightarrow \infty} \frac{m(r)^{2}}{r}=\lim _{r \rightarrow \infty} \frac{2 m(r)}{\sqrt{1+4 m^{2}(r)}}=1 .
$$


By l'Hopital's rule, (3-10) and (3-11) we have

$$
\begin{aligned}
\lim _{\rho \rightarrow+0} \rho \int_{a_{1}}^{\rho} \frac{1}{m^{2}(r)} d r=- & \lim _{\rho \rightarrow+0}\left(\frac{\rho}{m(\rho)}\right)^{2}=-1 . \\
\lim _{\rho \rightarrow+0} \rho \int_{a_{1}}^{i(q)-\rho} \frac{1}{m^{2}(r)} d r & =\lim _{\rho \rightarrow+0} \rho \int_{a_{1}}^{i(q)} \frac{1}{r} d r \\
& =\lim _{q \rightarrow p} d(p, q) \ln i(q)
\end{aligned}
$$

Note that $i(q)$ goes to infinity as $\rho \rightarrow+0$. By multiplying $\rho$ to the Equation (3-9) and taking a limit, we get

$$
\lim _{q \rightarrow p} d(p, q) \ln i(q)=1
$$

Corollary 3.3. Let $q$ be a point that is not a pole on the hyperboloid $z=\sqrt{1+x^{2}+y^{2}}$ and $\bar{q}$ the pole which is closest to $q$. Then

$$
\lim _{q \rightarrow \bar{q}} d(q, \bar{q}) i(q)
$$

is positive.

Proof: Let $q$ be any point that is not a pole. Then $i(q)$ satisfies $Y(i(q))=0$, where $Y(t)$ denotes the Jacobi field in Theorem 1. In our case,

$$
\int_{1}^{\infty} \frac{1}{m^{2}(t)} d t
$$

is finite, where $m$ satisfies the differential equation

$$
m^{\prime}(r)=\sqrt{\frac{1+m(r)^{2}}{1+2 m(r)^{2}}} \quad \text { with initial condition } \quad m(0)=0 .
$$

Hence by Theorem 3.1,

$$
c(L)-\int_{\rho}^{\infty} \frac{1}{L^{2}(r)} d r-\int_{i(q)-\rho}^{\infty} \frac{1}{L^{2}(r)} d r=0 .
$$

From Theorem 2.1 in [Tanaka, 92b], it follows that

$$
c(L)=\int_{r(M)}^{\infty} L(t)^{-2} d t
$$

where $r(M)=d(p, \bar{q})$. Thus by $(3-18)$,

$$
\int_{r(M)}^{\rho} \frac{1}{L^{2}(r)} d r=\int_{i(q)-\rho}^{\infty} \frac{1}{L^{2}(r)} d r .
$$

By L'Hopital's rule, we get

$$
\lim _{q \rightarrow \bar{q}}(i(q)-\rho) \int_{i(q)-\rho}^{\infty} \frac{1}{L^{2}(r)} d r=\left(\frac{1}{L^{\prime}(+\infty)}\right)^{2}=\frac{1}{2 \pi^{2}}
$$

Since $\rho-r(M)=d(q, \bar{q})$

$$
\begin{aligned}
\lim _{q \rightarrow \bar{q}}(i(q)-\rho) \int_{r(M)}^{\rho} \frac{1}{L^{2}(r)} d r & =\lim _{q \rightarrow \bar{q}} i(q) \int_{r(M)}^{\rho} \frac{1}{L^{2}(r)} d r \\
& =\frac{1}{L^{2}(r(M))} \lim _{q \rightarrow \bar{q}} i(q) d(q, \bar{q}) .
\end{aligned}
$$

By multiplying $i(q)-\rho$ to the Equation (3-20),

$$
\frac{1}{L^{2}(r(M))} \lim _{q \rightarrow \bar{q}} i(q) d(q, \bar{q})=\frac{1}{2 \pi^{2}} .
$$

Thus

$$
\lim _{q \rightarrow \bar{q}} i(q) d(q, \bar{q})=\frac{1}{2 \pi^{2}} L^{2}(r(M))=2 m(r(M))^{2}
$$

is positive.

\subsection{Asymptotic Scaling}

Estimating the error of our algorithm is not an easy matter, despite the fact that the numerical integration of the geodesic equations is standard. The reason is that the influence of the finite values of $N$ and $d_{\max }$ is not obvious.

The finite value of $d_{\max }$ produces points which are not poles, because geodesic curves proceeding from them intersect at a distance greater than $d_{\text {max }}$, therefore being incorrectly labeled as poles.

In the case of a rotationally symmetric two-sheeted hyperboloid (in our case $p=1$ ), we proved in Corollary 3.3 that the distance to the first (nearest) intersection of geodesic curves from a point $q$ which is not a pole (this point's injectivity radius $i(q))$ is asymptotically inversely proportional to this point's distance to the nearest pole (asymptotic in the sense that the inverse proportionality becomes more apparent the closer the two points are). We know that the projection of the set of poles of this 
surface onto the $(x, y)$-plane is a circle centered on the origin. A consequence of the asymptotic scaling is that we expect the algorithm to compute a radius which is too large by an amount which is inversely proportional to $d_{\max }$, given that $N$ is large enough. This is discussed further in Section 4.1.

We do not have a similar proven result for asymptotic scaling relating to $N$, but experimentally we find that the error in the radius of the projection of the set of poles for $p=1$ is inversely proportional to the square of $N$. See Section 4.1 for details.

\section{INDEPENDENT CHECKS}

In the following, we will use the few quantitative results known to independently check the output of our visualization program.

\subsection{The Radius of the Set of Poles for $\mathbf{p}=\mathbf{1}$.}

In Section III of his paper, von Mangoldt went into great detail in proving the statement that the set of poles of the surface given by (1-1) with $p=1$ includes the Scheitel and is bounded by a circle centered at this point. We wish to compute the radius of this circle using such methods as von Mangoldt provided, and use this to check the accuracy of the visualization program. We base our discussion on the explicit determination of this radius in Theorem 2.1 of [Tanaka, 92b], since this is much less involved than von Mangoldt's derivation.

We first compute the number $c(L)$ defined by $(3-5)$, where $L(t)$ is the length of the curve composed of points at a distance $t$ (arc length) from the Scheitel, and then (assuming that $c(L)$ is positive) the unique zero point of the function

$$
F(r)=c(L)-\int_{r}^{\infty} \frac{1}{L^{2}(s)} d s,
$$

which is the radius (in arc length from the Scheitel) of the set of poles.

On a two-sheeted rotationally symmetric hyperboloid, the curve of points a distance $t$ from the Scheitel is a circle, and its length $L$ is $2 \pi$ times the Euclidean distance in $\mathbb{R}^{3}$ from any of its points to the axis of symmetry. Due to rotational symmetry, we can restrict ourselves to the plane given by $y=0$, which the surface intersects at the curve given by $z=\sqrt{1+x^{2}}$. Given an arc length $t$, we can compute the $x$-coordinate of a point on this curve at the distance $t$ along the curve from the Scheitel, naming this coordinate $m(t)$, and we have $L(t)=2 \pi m(t)$, as in Equation (3-6). In practice, the function $m(t)$ can be computed as the solution of the ordinary differential equation given by $(3-17)$.

We obtain

$$
c(L) \approx 0.0360682470577406
$$

and the radius

$$
r \approx 1.016650084
$$

for which

$$
m(r) \approx 0.931802372 .
$$

This last number is the radius of the projection of the set of poles onto the $(x, y)$-plane. The projection is a disk centred at $(0,0)$. The visualization routines produce the value of

$$
\tilde{m}(r) \approx 0.931802654
$$

Now we are in a position to discuss the asymptotic scaling laws for our algorithm's error in computing the set of poles of this surface $(p=1)$.

First we use Corollary 3.3 and check whether the error in $m(r)$ is asymptotically inversely proportional to $d_{\max }$, given a large enough value of $N$. In our experiments, we used the radius computed above (Equation (4-4)) as the exact result. We concluded that we are indeed approaching asymptotic scaling, but that the effect of the finite value of $N$ is significant.

Given the value of $m(r)$ (Equation (4-4)), we can compute the value of the constant on the right hand side of (3-24). We must remember that the data correspond to distances and errors in distances computed in the projection onto the $(x, y)$-plane. Correcting for this, we find that the constant should have the value of approximately 1.015. This is indeed consistent with our data. It is difficult to say more due to the effect of the finite value of $N$.

One can of course also investigate the effect of varying $N$ for a given, large value of $d_{\max }$ (i.e. $10^{50}$ ). If we extrapolate to $1 / N=0$, we find

$$
m(r) \approx 0.931802371
$$

which is in excellent agreement with (4-4). The data strongly suggest scaling of the form

$$
\tilde{m}(r) \approx m(r)+\frac{\text { const }}{N^{2}}
$$

for large $d_{\text {max }}$, but we have not been able to prove this. 

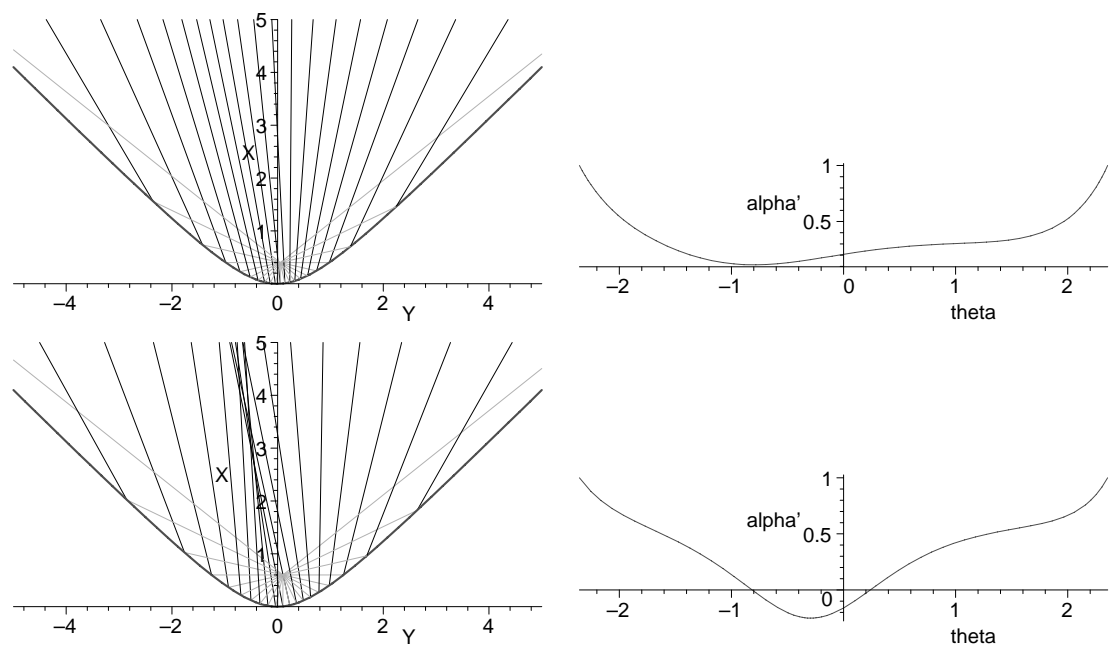

FIGURE 2. $p=\infty$ : The surface (1-1) has become two flat pieces, each bounded by a hyperbola and joined at this common boundary. The two illustrations on the left show geodesic curves from the points $(0.4,0.05)$ and $(0.6,0.1)$, the upper and lower plots respectively. These curves are grey on the same flat piece as their source, and black after they have reflected and are on the other piece. The upper plot corresponds to a pole, since none of the geodesic curves intersect other than at their source. The lower plot corresponds to a point which is not a pole. The two illustrations on the right show the rate of change of the angle of the black geodesic curves as a function of the angle at which they left their source on the other side of the surface. A source point is not a pole if this rate can be negative.

\subsection{The Values of $p$ for which the Scheitel is a Pole}

In Section V of von Mangoldt's paper, he proved that one can make the Scheitel cease being a pole by increasing $p$ beyond 1 . He proved the existence of a critical value of $p$ without explicitly computing it, but did provide the necessary equations (the Jacobi equation applied to the curve $\gamma$, which we define as the intersection of the surface given by $(1-1)$ and the plane given by $x=0)$. We get the estimate

$$
p_{c} \approx 4.856874
$$

which corresponds well with the estimate derived from the visualization routines, which is

$$
\tilde{p}_{c} \approx 4.85688
$$

\subsection{Some Educated Guesswork concerning $\mathbf{p}=\infty$}

This Section will not contain any proofs, but details of a computation which we feel supports one of the conjectures we will formulate later.

As $p$ tends towards infinity, the surface (1-1) becomes more and more like two identical flat pieces joined at their common boundary (the curve given by $x=0$ and $z=$ $\left.\sqrt{1+y^{2}}\right)$. We choose to parametrize each piece using $(X, Y)$-coordinates, where

$$
(X, Y)=(z-1, y)
$$

Note that $X \geq \sqrt{1+Y^{2}}-1$, and that the coordinates of the Scheitel are $(0,0)$. These coordinates have the advantage that the distance from the Scheitel to any point on either flat piece is simply $\sqrt{X^{2}+Y^{2}}$. For the moment we are assuming that $X \geq 0$, but later on we will see that it makes sense to indicate which piece one is on by using the sign of $X$.

Geodesic curves on this surface are straight lines until they reach the boundary of one of the pieces. At the boundary, geodesic curves change from the one flat piece to the other, satisfying the usual law of reflection at a flat surface (angle of reflection equals angle of incidence). Two geodesic curves can only intersect on the same flat piece (i.e. on the same side of the surface). See Figure 2.

Given a source point with coordinates $\left(X_{0}, Y_{0}\right)$ on one side of the surface (and not on the boundary), and a unit speed geodesic $\gamma$ such that $\gamma(0)=\left(X_{0}, Y_{0}\right)$ and $\gamma^{\prime}(0)=$ $(-\cos \theta, \sin \theta)$, we label the first point of contact with the boundary $\left(X_{1}, Y_{1}\right)$. We can compute a point $\left(X_{2}, Y_{2}\right)$ on the continuation of this geodesic curve on the other side (not on the boundary) after reflection.

Now we are in the position to define an algorithm. This algorithm will not be perfect, but its weaknesses will be different than those of the visualization software used in the remainder of the paper, so its results are of interest in so far as they agree with others we present. The idea is to measure the angle of the geodesic curves with respect to the $X$-axis after reflection (the black lines 


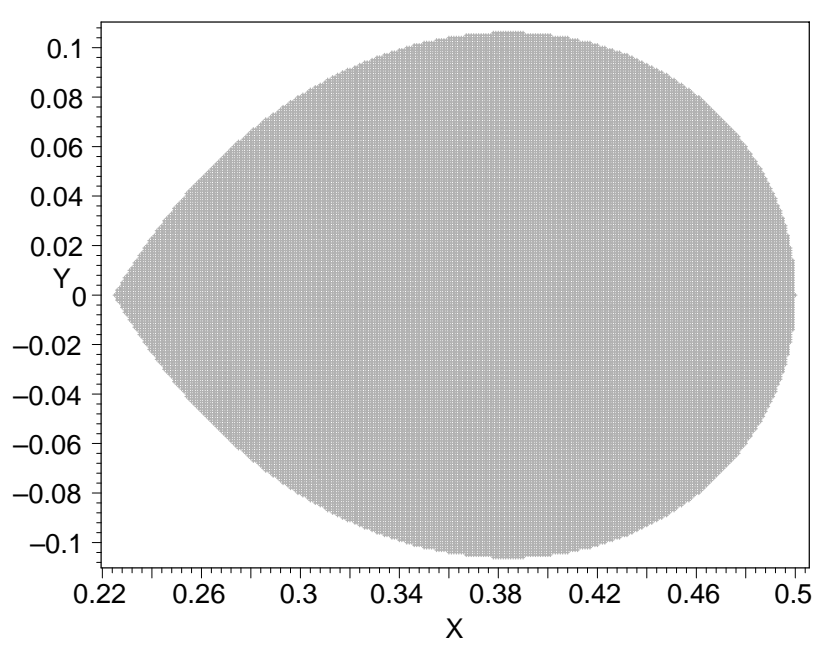

FIGURE 3. One component of the set of poles for $p=\infty$.

in Figure 2), and consider this a function of the angle $\theta$ at which they left the source point. We ignore the possibility that a geodesic curve can reflect many times because the source points we are interested in studying do not give rise to this. If we define the outgoing angle as

$$
\alpha=\tan ^{-1} \frac{Y_{2}-Y_{1}}{X_{2}-X_{1}}
$$

then we can study the rate of change of $\alpha$ as a function of $\theta$. If $\alpha$ is strictly monotone increasing, then we label the source point a pole, otherwise not. The plots on the right hand side of Figure 2 are of $\alpha^{\prime}(\theta)$. If we use this algorithm to look for poles within $\left(X_{0}, Y_{0}\right) \in[0.2,0.6] \times[-0.3,0.3]$, then we find the set depicted in Figure 3.

Of course both sides of the surface will have such a set. We show only one component of the set of poles of the surface, since the other component is identical to it in every way except that it is on the other flat piece. One can in fact consider the surface to be the set

$$
M=\left\{(X, Y):|X| \geq \sqrt{1+Y^{2}}-1\right\}
$$

where the boundary points $\left(\sqrt{1+Y^{2}}-1, Y\right)$ and $(1-$ $\left.\sqrt{1+Y^{2}}, Y\right)$ are to be identified for all $Y$.

The check consists of comparing this set with results for very large $p$. This will be done in Section 5.1, where the $(X, Y)$-coordinates used here are discussed again. We can however already state that the comparison is extremely favourable, and encourage the reader to look ahead to Figure 8.

\subsection{The Set of Poles of a Paraboloid}

In this section, we apply the visualization software to treat the surface given by

$$
z=x^{2}+y^{2} \text {. }
$$

In Section IV of von Mangoldt's paper, he proved that the set of poles consists only of the Scheitel $(0,0,0)$, which is the same point as the vertex of this surface.

The proof of the asymptotic scaling law used in the hyperbolic case does not apply here. Instead, we know (Corollary 3.2) that the logarithm of the distance to the first (nearest) intersection of geodesic curves from a point which is not a pole (this point's injectivity radius) is asymptotically inversely proportional to this point's distance to the vertex. This means that convergence to correct results is asymptotically proportional to $1 / \log \left(d_{\max }^{2}\right)$ or, equivalently, $1 / \log d_{\max }$, rather than $1 / d_{\text {max }}$, making the paraboloid more difficult to treat than a hyperboloid. The square in $1 / \log \left(d_{\text {max }}^{2}\right)$ is due to the fact that $d_{\max }$ measures Euclidean distance in the projection onto the $(x, y)$-plane rather than distance along a geodesic on the surface.

The check consists of using our visualization routines to find the radius of the set of poles of the paraboloid, which we already know to be zero. We did indeed find results consistent with the radius going to zero as $d_{\max }$ goes to infinity. Changing the value of $N$ (down to 4096) was found to have an almost insignificant (but otherwise not surprising) effect.

\section{RESULTS FOR THE TWO-SHEETED HYPERBOLOID}

For $p=0$, we know that all points are poles. Also, the umbilic points are always poles. In the limit $p \rightarrow \infty$, the coordinates of the two umbilic points tend to $( \pm 0,0, \sqrt{2})$. The output of the visualization routines is in agreement with these facts.

Furthermore, the breaking of the set of poles into two disjoint sets for large enough $p$ already predicted by von Mangoldt is confirmed (see Figure 4).

What one notices when looking at the set of poles for very small $p$ (see Figure 5 ) is that the set of poles is indeed large, giving one reason to believe that there is a continuous transition from small, finite sets for $p \approx 1$ to larger and larger sets as $p$ decreases, until the entire infinite surface is included for $p=0$.

Figure 6 supports this picture, and suggests further that the set of poles for a surface given by some value of $p$ contains the sets of poles for other surfaces corresponding to larger values of $p$. We need to define what we mean by 

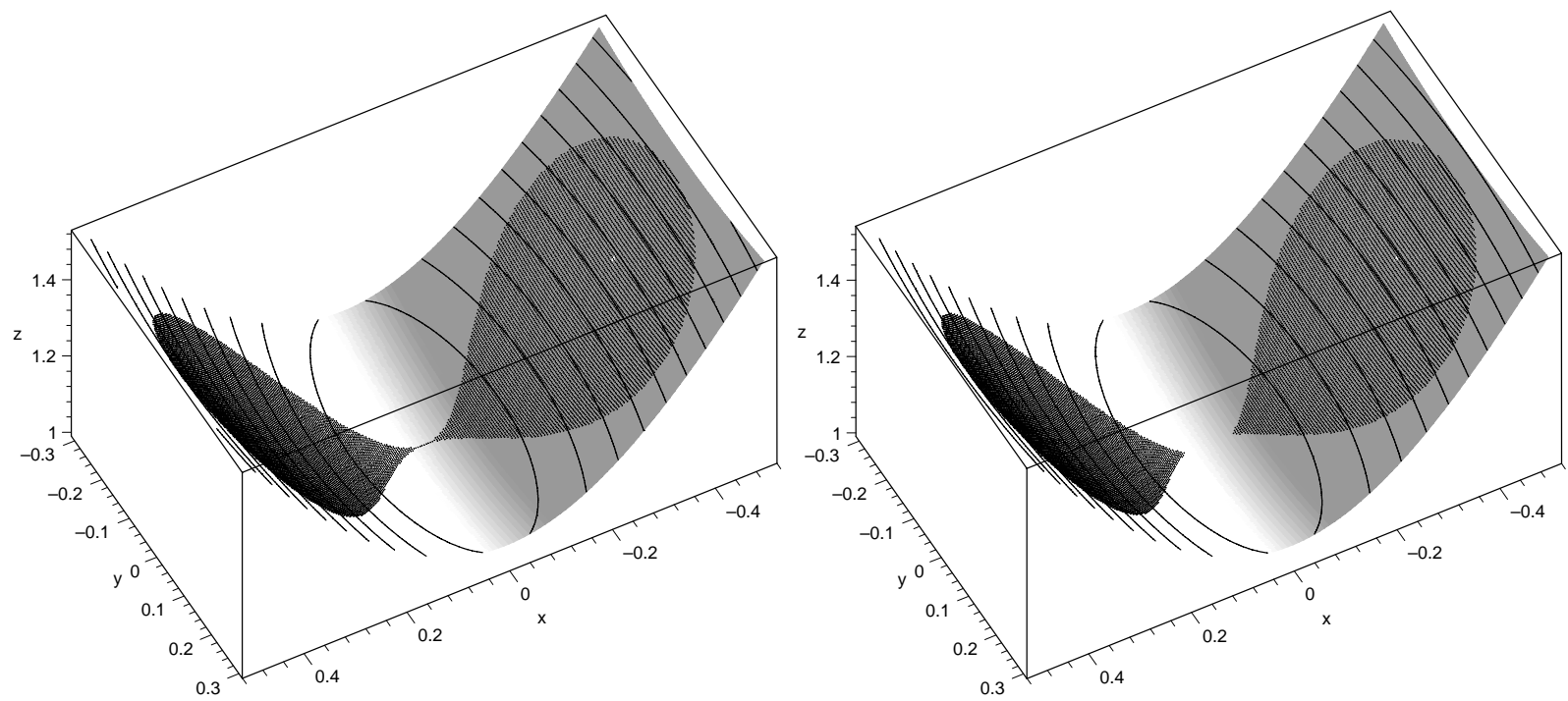

FIGURE 4. The set of poles for $p=4.85687918$ (above) and $p=5$ (below) on the surfaces given by $z=\sqrt{1+p x^{2}+y^{2}}$. The former is less than but very close to the critical value of $p_{c} \approx 4.856874$. The latter is larger than the critical value, and the set of poles has broken into two disjoint subsets.
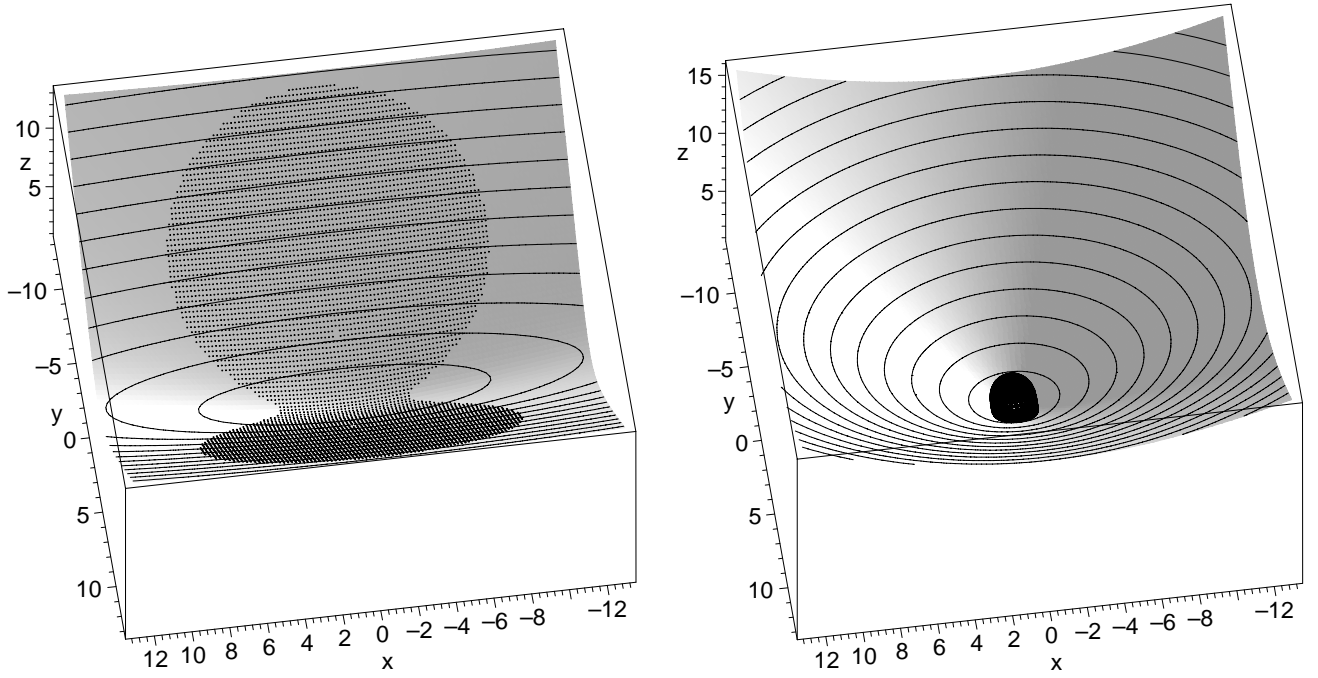

FIGURE 5. The set of poles for $p=0.05$ (left) and $p=0.5$ (right).

"contains", because poles are points on their respective surfaces, and these surfaces only intersect at the curve given by $x=0$ and $z=\sqrt{1+y^{2}}$. Our results suggest that the most useful formulation makes use of geodesic polar coordinates, and we use these in the following. All calculations of geodesic polar coordinates were performed using a slightly modified version of the software package Loki [Sinclair and Tanaka, 02].

\subsection{Conjectures}

For any given value of $p \geq 0$, compute the set of poles of the surface given by $z=\sqrt{1+p x^{2}+y^{2}}$. For each pole $q$ which is not the Scheitel, compute its geodesic polar coordinates $(r, \theta)$ around the Scheitel, by finding the unique unit speed geodesic $\gamma_{q}$ emanating from the Scheitel and passing through the pole $q$, such that $\gamma_{q}(0)=(0,0,1)$ (the Scheitel), defining $r$ by $\gamma_{q}(r)=q$ and $\theta$ by $(\cos \theta, \sin \theta, 0)=\gamma_{q}^{\prime}(0)$.

Then compute each pole's $(X, Y)$-coordinates, where $X=r \cos \theta$ and $Y=r \sin \theta$. The Scheitel itself is mapped to $\left(X_{S}, Y_{S}\right)=(0,0)$. We now have a projection from the set of poles of a surface for some given value of $p$ to $\mathbb{R}^{2}$. Let us call these sets in $\mathbb{R}^{2} \mathcal{P}(p)$. Then our results, especially Figures 6 and 7, suggest very strongly that 


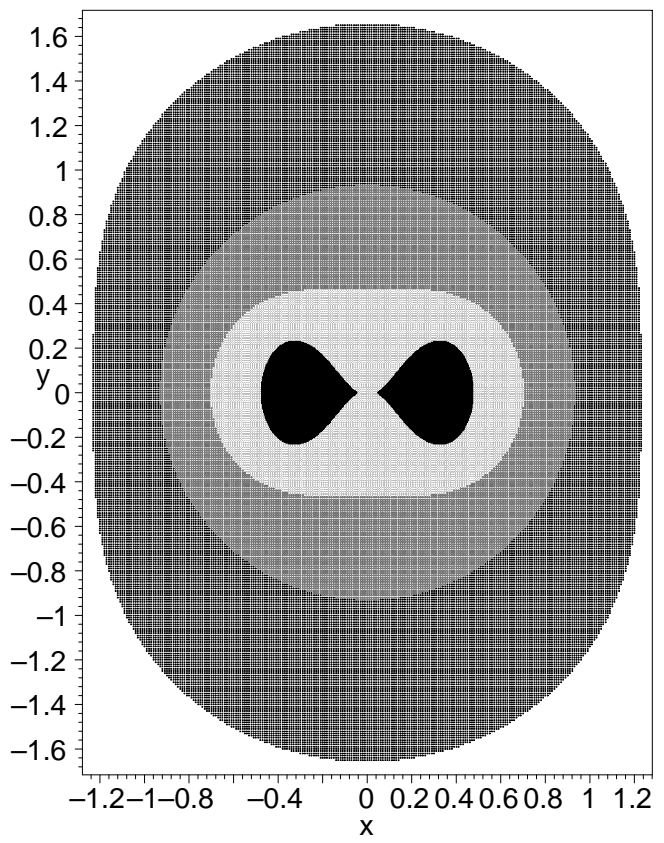

FIGURE 6. The projections onto the $(x, y)$-plane of the sets of poles for $p=0.5,1,2$ and 5 . The set for $p=0.5$ includes all the black regions and their interiors. The set for $p=1$ is a circular disc centred at the origin; it is the grey region and its interior. The set for $p=2$ is the white region and its interior. The set for $p=5$ consists of the two disjoint tear-shaped sets in the middle of the figure. Note that each set contains the next.

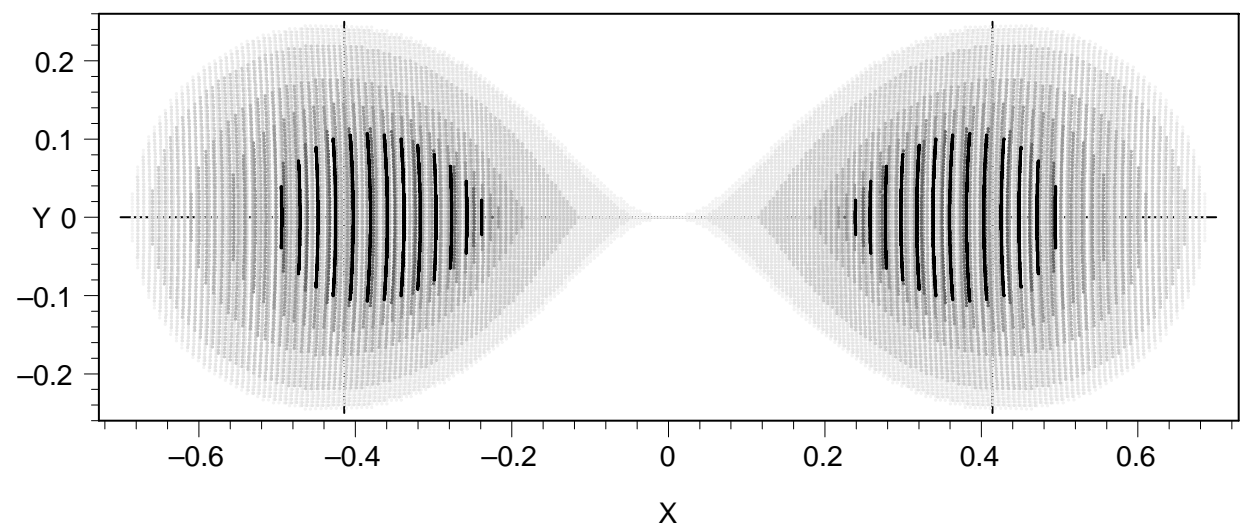

FIGURE 7. The set of poles for $p=4.85687918$ (light grey), 5, 6, 10, 20, 100 and 1000 (black). What one should notice is that each set contains the next. The candidate points were first chosen from a regular grid on the projection of each surface onto the $(x, y)$-plane, and the stripes in the figure are an artifact of this grid. Then geodesic polar coordinates $(r, \theta)$ around the Scheitel were computed for each pole, and finally each one was plotted in $(X, Y)$-coordinates, where $X=r \cos \theta$ and $Y=r \sin \theta$.

$$
\mathcal{P}\left(p_{1}\right) \subset \mathcal{P}\left(p_{2}\right) \quad \Longleftrightarrow \quad p_{1}>p_{2} .
$$

Finally, we already know that

$$
( \pm[\sqrt{2}-1], 0) \in \lim _{p \rightarrow \infty} \mathcal{P}(p)
$$

(if $\lim _{p \rightarrow \infty} \mathcal{P}(p)$ exists) because the umbilic points are always poles, but Figures 7 and 8 suggest that $\lim _{p \rightarrow \infty} \mathcal{P}(p)$ contains more than just these two points. We claim this because $\mathcal{P}\left(10^{3}\right)$ appears to the eye to be identical to $\mathcal{P}\left(10^{4}\right)$. The limiting set would therefore seem to be that depicted in Figure 8.

It is at this point that we can return to the experiments performed in Section 4.3. The $(X, Y)$ coordinates used in that Section correspond in every way to those used here, and allow a direct comparison of Figures 3 and 8 . One can immediately see that they are essentially identical (of course we expect 


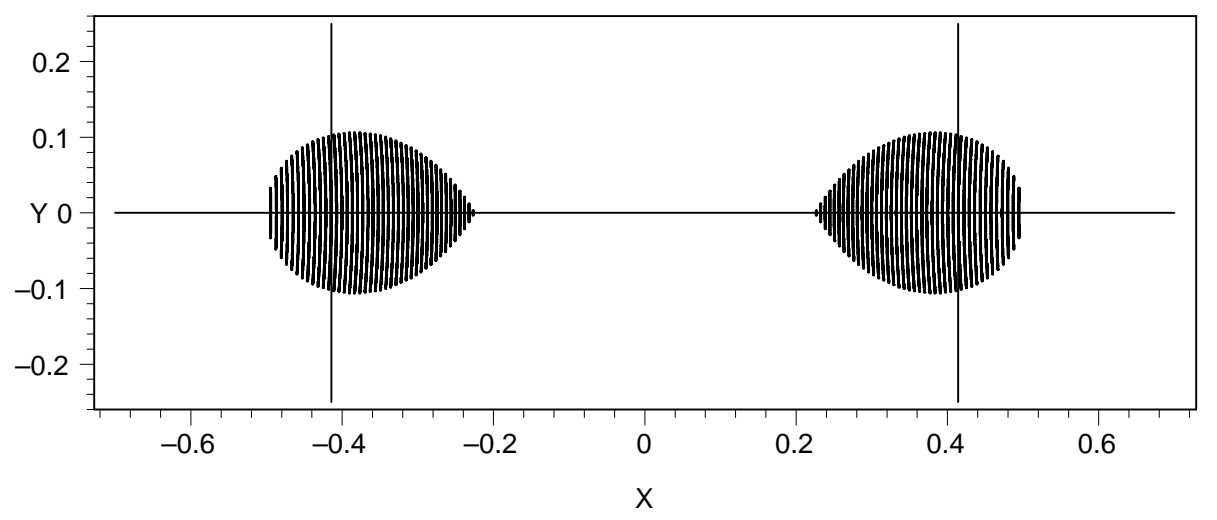

FIGURE 8. The set of poles for $p=10000$. See the caption of Figure 7 for an explanation of the coordinates used. The horizontal and vertical lines mark the limiting positions of the umbilic points as $p \rightarrow \infty$.

some difference because $10^{4} \neq \infty$, but when one plots them together on the same axes no difference is visible). This excellent match is the primary motivation for our second conjecture - that $\lim _{p \rightarrow \infty} \mathcal{P}(p)$ exists and is the set shown in Figures 8 and 3 .

\section{CONCLUSION}

We have provided the first quantitatively reliable visualizations of the set of poles of a family of two-sheeted hyperboloids, capturing a process previously only qualitatively described, and making further conjectures.

From a computational point of view, the most interesting result of this investigation has been the role of differential geometry in the error analysis of our algorithm via asymptotic scaling laws. This demonstrates once again the mutual interdependence of computational and traditional mathematics.

\section{ACKNOWLEDGEMENTS}

R. Sinclair was supported in his stay in Japan by a grant from Otto Mønsteds Fond.

\section{REFERENCES}

[Arnold, 1994] V.I. Arnold, Topological Invariants of Plane Curves and Caustics, University Lecture Series, Vol. 5, AMS, Providence, Rhode Island (1994).

[Berger, 00] M. Berger. Riemannian geometry during the second half of the twentieth century, University Lecture Series, Vol. 17, AMS, Providence, Rhode Island, 2000.

[von Braunmühl, 1882] A. v. Braunmühl. "Geodätische Linien und ihre Enveloppen auf Dreiaxigen Flächen Zweiten Grades." Math. Annalen 14 (1882), 557-586.

[Chavel, 93] I. Chavel. Riemannian Geometry: A Modern Introduction, Cambridge University Press, New YorkMelbourne, 1993.

[von Mangoldt, 1881] H. v. Mangoldt. "Ueber diejenigen Punkte auf positiv gekrümmten Flächen, welche die Eigenschaft haben, dass die von ihnen ausgehenden geodätischen Linien nie aufhören, kürzeste Linien zu sein." $J$. für die reine und angewandte Mathematik 91:1 (1881), 23-53.

[Tanaka, 92a] M. Tanaka. "On the Cut Loci of a von Mangoldt's Surface of Revolution." J. Math. Society of Japan. 44:4 (1992), 631-641.

[Tanaka, 92b] M. Tanaka. "On a characterization of a surface of revolution with many poles." Memoirs of the Faculty of Science, Kyushu University, Ser. A. 46: 2 (1992), 251-268.

[Sinclair and Tanaka, 02] R. Sinclair and M. Tanaka. "Loki: Software for Computing Cut-Loci." Experimental Mathematics 11:1 (2002)

Robert Sinclair, Department of Mathematics \& Statistics, The University of Melbourne, Parkville, Victoria 3010, Australia (sinclair@ms.unimelb.edu.au)

Minoru Tanaka, Department of Mathematics, Tokai University, Hiratsuka City, Kanagawa Pref. 259-1292, Japan

(m-tanaka@sm.u-tokai.ac.jp

Received December 17, 2000; accepted in revised form July 16, 2001. 